\title{
AN RBF INTERPOLATION BLENDING SCHEME FOR EFFECTIVE SHOCK-CAPTURING
}

\author{
M. HARRIS ${ }^{1}$, A. KASSAB ${ }^{1} \&$ E. DIVO ${ }^{2}$ \\ ${ }^{1}$ Department of Mechanical and Aerospace Engineering, University of Central Florida, Orlando FL USA. \\ ${ }^{2}$ Department of Mechanical Engineering, Embry-Riddle Aeronautical University, Daytona Beach, FL USA.
}

\begin{abstract}
In recent years significant focus has been given to the study of Radial basis functions (RBF), especially in their use on solving partial differential equations (PDE). RBF have an impressive capability of interpolating scattered data, even when this data presents localized discontinuities. However, for infinitely smooth RBF such as the Multiquadrics, inverse Multiquadrics, and Gaussian, the shape parameter must be chosen properly to obtain accurate approximations while avoiding ill-conditioning of the interpolating matrices. The optimum shape parameter can vary significantly depending on the field, particularly in locations of steep gradients, shocks, or discontinuities. Typically, the shape parameter is chosen to be high value to render flatter RBF therefore yielding a high condition number for the ensuing interpolation matrix. However, this optimization strategy fails for problems that present steep gradients, shocks or discontinuities. Instead, in such cases, the optimal interpolation occurs when the shape parameter is chosen to be low in order to render steeper RBF therefore yielding low condition number for the interpolation matrix. The focus of this work is to demonstrate the use of RBF interpolation to capture the behaviour of steep gradients and shocks by implementing a blending scheme that combines high and low shape parameters. A formulation of the RBF blending interpolation scheme along with testing and validation through its implementation in the solution of the Burger's linear advection equation and compressible Euler equations using a Localized RBF Collocation Meshless Method (LRC-MM) is presented in this paper.
\end{abstract}

Keywords: compressible flow, meshless, multiquadrics, radial basis function, RBF, shock.

\section{INTRODUCTION}

The governing equations found in science and engineering involve partial differential equations (PDE) which can be difficult to solve and most require numerical methods. Solutions to PDE exist for linear and simple geometry problems, but in engineering coupled, non-linear PDE with complex geometry are often encountered and must be solved numerically. These complexities can be seen with the Navier-Stokes equations. Current numerical methods make use of the Finite Difference Methods (FDM), Finite Element Method (FEM) and more commonly in CFD the Finite Volume Method (FVM). These mesh based methods are well developed numerical techniques and are the methods used today for solving PDEs [1-10]. Mesh based methods require a mesh, grid, or connectivity to be defined within the domain so the governing PDEs can be discretized and solved. The mesh or connectivity between nodes must be predetermined before attempting a solution. The mesh quality can cause issues with convergence as well. Developing a high quality mesh having both the resolution to capture the physics of the problem while also minimizing the number of cells, and thus minimizing computational time, can be an exhausting iterative process. For very complex problems, one could conclude this task to be impossible and must seek simplifying assumptions or accept the solution. Automated mesh generators have been developed to alleviate the process required to produce a good quality mesh in very little time. However, automated mesh generators do not necessarily guarantee a good mesh and are far from being fully automated. The user is required to estimate sizes, generate the mesh and inquire if the mesh has met an acceptable criterion possibly having to re-mesh if it is unacceptable. 
Meshless methods have been an area of research and development in recent years. These techniques originate from spectral methods based on Legendre or Chebyshev polynomial, which require uniform point distribution [8-10]. However, the meshless methods using radial basis functions (RBF) can be used on non-uniform distributions of points. The RBFs are based on the Euclidean distance and RBF such as the Hardy multiquadrics and inverse Hardy multiquadrics are depend on the shape parameter, c. RBF meshless methods depend on local or global interpolation on irregular spatial distribution of points and not connectivity of points providing a solution to the complexity of mesh generation. The absence of the connection between points is how meshless methods facilitate the solution process, without the need to develop a mesh only requiring only a point cloud and boundary nodes to solve.

Many meshless methods exist, but the focus of this work involves methods using radial basis (RBF) interpolation. Interpolation using RBF has shown to provide spectral accuracy, but while using the Hardy multiquadrics and inverse multiquadrics, the accuracy is dependent on the shape parameter, $\mathrm{c}$. The shape parameter is either found by numerical experimentation or is arbitrarily chosen to determine the best value. It is often found that a higher valued shape parameter causing the interpolation matrix to become close to ill-conditioned gives the best accuracy $[1,2]$. The issue of the ill-conditioning does not allow for global interpolation as solutions are not accurate, but researchers have found that locally interpolating using RBF methods resolves these issues $[2,4,7,8,10]$.

The shape parameter being a large value causing the condition number of the interpolation matrix to be large allows for convergence for smooth functions [1, 10]. The shape parameter value needed is usually arbitrarily chosen to be large throughout the solution [2]. This is not the case in the presence of steep gradient or highly convective flows. Oscillations of the solution tend to manifest using the approach of large shape parameter RBF interpolation. It happens to be that a low shape parameter value causing the conditioning of the interpolation matrix to be low tends to provide better accuracy for functions with steep gradients. So if one must solve regions where the field is smooth while steep gradients or discontinuities can exist downstream, the RBF interpolation must be blended between high and low shape parameter values.

This work discusses the RBF interpolation of smooth and steep gradient functions demonstrating how the shape parameter can change the accuracy. The blended RBF interpolation formulation is described showing the approach taken to sense steep gradients then change the shape parameter from a high value to low based on the curvature of the field. Finally, how well the interpolation is capable of capturing the discontinuity is presented by showing examples of solutions to the inviscid Burgers equation, 2-D linear advection equation and the 2-D compressible Euler equations.

\section{RBF INTERPOLATION OVERVIEW}

Interpolation using a RBF is implemented by assuming the function is equal to the summation described by

$$
f(x, y)=\sum_{j=1}^{N} \alpha_{j} \chi_{j}(x, y)
$$

where $\alpha_{j}$ are the coefficients, $\chi_{j}(x, y)$ is the expansion function and $N$ is the number of collocation points. The coefficients are determined through a collocation process of the function $f(x, y)$ at the collocation points, $f\left(x_{i}, y_{i}\right)$ for $i=1 \ldots N$. 


$$
f\left(x_{i}, y_{i}\right)=\sum_{j=1}^{N} a_{j} \chi_{j}\left(x_{i}, y_{i}\right)
$$

A system of equations can be formed and the equations can be presented in matrix form as

$$
\{f\}=[C]\{\alpha\}
$$

where $[\mathrm{C}]$ is of the form

$$
[C]=\left[\begin{array}{ccc}
\chi_{1}\left(x_{1}, y_{1}\right) & \ldots & \chi_{N}\left(x_{1}, y_{1}\right) \\
\vdots & \ddots & \vdots \\
\chi_{1}\left(x_{N}, y_{N}\right) & \cdots & \chi_{N}\left(x_{N}, y_{N}\right)
\end{array}\right]
$$

The coefficients, $a_{j}$, are then found by solving the linear system of equations by

$$
\{a\}=[C]^{-1}\{f\}
$$

The function or field variable can now be solved by

$$
\begin{gathered}
f(x, y)=\{\chi(x, y)\}^{T}\{\alpha\} \\
f(x, y)=\{\chi(x, y)\}^{T}[C]^{-1}\{f\}
\end{gathered}
$$

Similarly, the derivative of the function can be found by

$$
\begin{aligned}
& \frac{\partial f(x, y)}{\partial x}=\left\{\frac{\partial \chi(x, y)}{\partial x}\right\}^{T}[C]^{-1}\{f\} \\
& \frac{\partial f(x, y)}{\partial y}=\left\{\frac{\partial \chi(x, y)}{\partial y}\right\}^{T}[C]^{-1}\{f\}
\end{aligned}
$$

The expansion functions, $\chi_{j}(x, y)$, are chosen to be a RBF. Many RBFs exist, but for the scope of this paper only the Hardy Multiquadrics and the Inverse Multiquadrics are considered. Example of these functions are

Hardy Multiquadrics RBF: $\quad \chi_{j}(x, y)=\sqrt{\left[r_{j}^{2}(x, y)+c^{2}\right]}$

Inverse Multiquadrics RBF: $\quad \chi_{j}(x, y)=\frac{1}{\sqrt{\left[r_{j}^{2}(x, y)+c^{2}\right]}}$

These RBFs are dependent on the shape parameter, $c$, which has a significant effect on the behaviour of the approximation of the function and its derivatives. This shape parameter is typically chosen to be a large value so that the matrix $[C]$ is of high conditioning. In the following sections, it is shown this methodology fails for problems with steep gradients, highly convective flows, and when shocks are encountered. 


\section{RBF INTERPOLATION OF SMOOTH AND DISCONTINUOUS FUNCTIONS}

As mentioned before, the shape parameter, $c$, is chosen to cause the interpolation matrix approach a high condition number, almost to the edge of ill-conditioning. This approach is the appropriate method for smooth functions, but this is not the case for regions of highly convective flows, steep gradients, or discontinuities. Actually, a shape parameter value providing lower conditioning of the interpolation matrix provides better results for steep gradients. This result is further described in this section. The shape parameter is varied using values of $\mathrm{c}=0.1, \mathrm{c}=0.5$, and $\mathrm{c}=1.0$ for the RBF reproduction of the test function $f(x)=\tan ^{-1}\left[\omega\left(x-x_{0}\right)\right]$. The value of $\omega$ is varied to change the gradient of the test function. The RBF interpolation does very well at reproducing the smooth test function of $\omega=1$, but a higher shape parameter causing a high conditioning number for the interpolation matrix provides much lower $\mathrm{L}^{2}$-norm errors [12]. The errors for the smooth function interpolation are shown in Table 1. Next, the RBF interpolation is tested on the test function with $\omega=10$ and is shown in Figure 2.
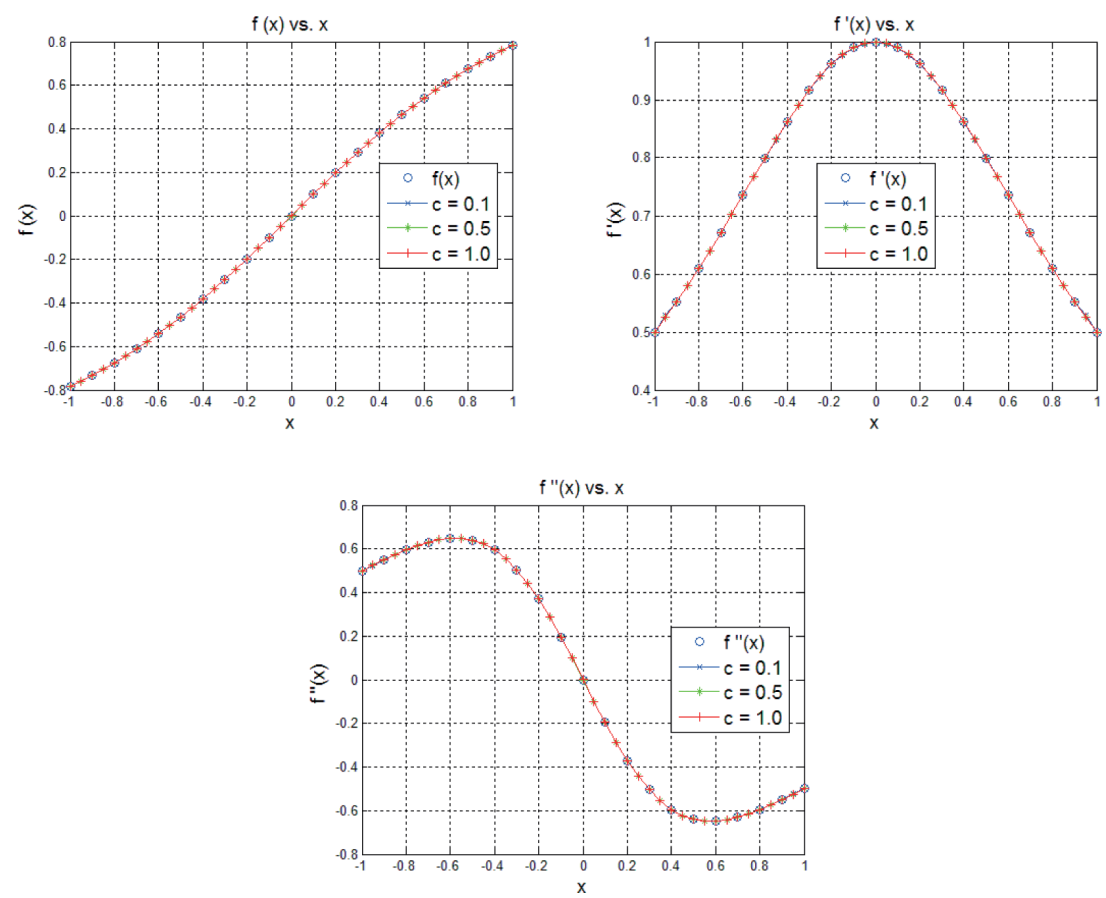

Figure 1: Interpolation of smooth function.

Table 1: $\mathrm{L}^{2}$ Norms for Smooth Function RBF Interpolation.

\begin{tabular}{lccc}
\hline $\mathrm{c}$ & $f(x)$ & $f^{\prime}(x)$ & $f^{\prime \prime}(x)$ \\
\hline 0.1 & $2.52 \mathrm{e}-4$ & 0.0016 & $3.46 \mathrm{e}-4$ \\
0.5 & $3.20 \mathrm{e}-6$ & $1.64 \mathrm{e}-5$ & $5.71 \mathrm{e}-6$ \\
1.0 & $1.41 \mathrm{e}-8$ & $4.41 \mathrm{e}-8$ & $1.45 \mathrm{e}-8$ \\
\hline
\end{tabular}



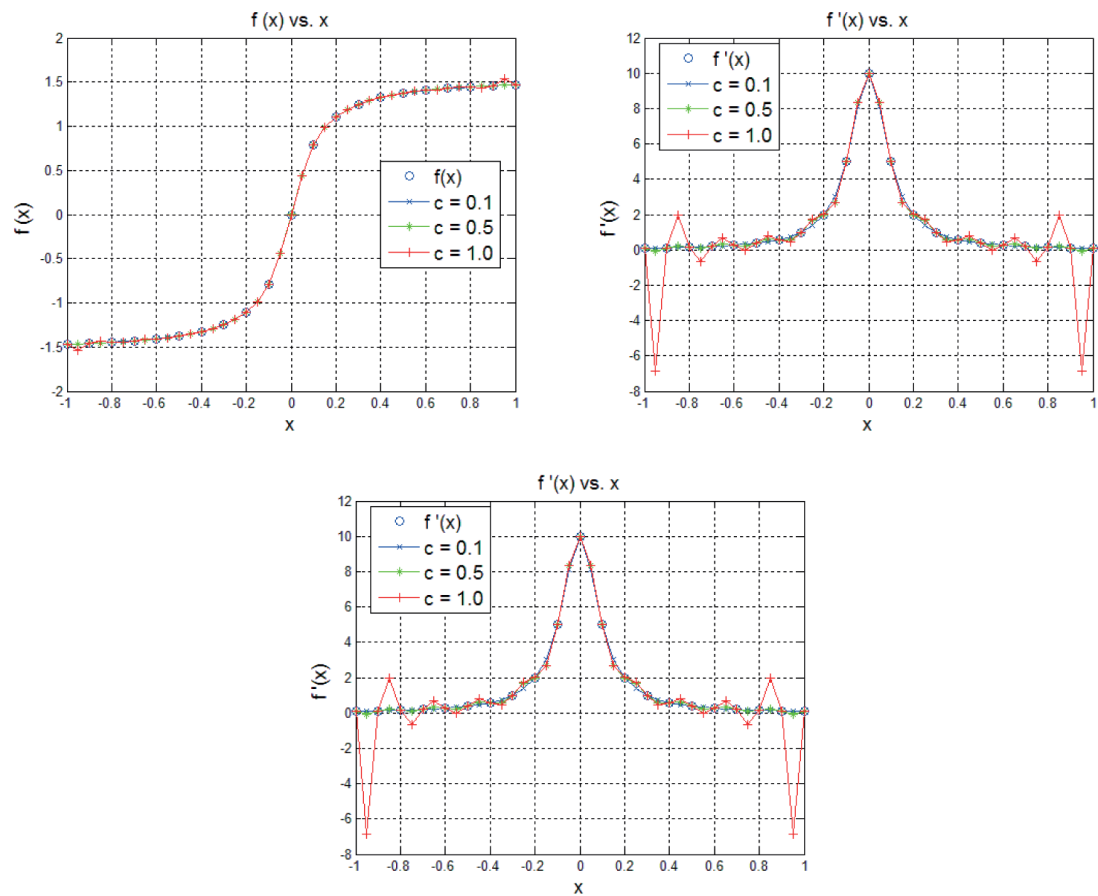

Figure 2: Interpolation of function with steep gradient.

Table 2: $\mathrm{L}^{2}$ Norm for function with Steep Gradient RBF Interpolation.

\begin{tabular}{lccc}
\hline $\mathrm{c}$ & $f(x)$ & $f^{\prime}(x)$ & $f^{\prime \prime}(x)$ \\
\hline 0.1 & 0.0021 & 0.0069 & 0.0526 \\
0.5 & 0.0017 & 0.0167 & 0.0480 \\
1.0 & 0.0060 & 0.2369 & 0.0810 \\
\hline
\end{tabular}

\section{BLENDED RBF INTERPOLATION SCHEME}

To take advantage of the RBF interpolation when encountering both smooth and steep gradients in the field, the RBF interpolation scheme must have the capability to blend between high and low values shape parameter. The shape parameter, c, could be adjusted during the computation but a relationship for, $\mathrm{c}$, is difficult to determine requiring time consuming numerical experimentation. A blended approach allows the user to set the shape parameter values for smooth and steep gradient interpolation and blend between the two interpolations as needed. We first assume the function $f_{c}(x, y)$ is in between the function $f_{a}(x, y)$ and $f_{b}(x, y)$ so that,

$$
f_{c}(x, y)=f_{a}+\phi\left(f_{b}-f_{a}\right)
$$


where $f_{a}$ in this case in the smooth function interpolation and $f_{b}$ the steep gradient interpolation.

$$
\begin{aligned}
& f_{a}(x, y)=\sum_{j=1}^{N} a_{a_{j}} \chi_{a_{j}}(x, y) \\
& f_{b}(x, y)=\sum_{j=1}^{N} a_{b_{j}} \chi_{b_{j}}(x, y)
\end{aligned}
$$

Defining two RBF with high and low valued shape parameters $c_{a}$ and $c_{b}$. The Hardy Multiquadrics $\mathrm{RBF}$ is used as an example,

$$
\begin{aligned}
& \chi_{a_{j}}(x, y)=\sqrt{\left(x-x_{j}\right)^{2}+\left(y-y_{j}\right)^{2}+c_{a}^{2}} \\
& \chi_{b_{j}}(x, y)=\sqrt{\left(x-x_{j}\right)^{2}+\left(y-y_{j}\right)^{2}+c_{b}^{2}}
\end{aligned}
$$

By introducing discrete points at locations $x_{i}$, the functions can be evaluated as

$$
\begin{aligned}
& f_{a}\left(x_{i}, y_{i}\right)=\sum_{j=1}^{N} a_{a_{j}} \chi_{a_{j}}\left(x_{i}, y_{i}\right) \\
& f_{b}\left(x_{i}, y_{i}\right)=\sum_{j=1}^{N} a_{b_{j}} \chi_{b_{j}}\left(x_{i}, y_{i}\right)
\end{aligned}
$$

Next, the weights $\alpha_{a_{j}}$ and $a_{b_{j}}$ must be determined. Equations (12) and (13) are used to form a system of equations using the scattered data of $f\left(x_{i}, y_{i}\right)$.

$$
\begin{aligned}
& {\left[\chi_{a}\right]\left\{a_{a}\right\}=\{f\}} \\
& {\left[\chi_{b}\right]\left\{a_{b}\right\}=\{f\}}
\end{aligned}
$$

Inverting the interpolation matrices $\left[\Psi_{a}\right]$ and $\left[\Psi_{b}\right]$ and solving for the weighting coefficients

$$
\begin{aligned}
& \left\{a_{a}\right\}=\left[\chi_{a}\right]^{-1}\{f\} \\
& \left\{a_{b}\right\}=\left[\chi_{b}\right]^{-1}\{f\}
\end{aligned}
$$

Substituting equations (21) and (22) into (12) we have the blended expression.

$$
f_{c}(x, y)=\sum_{j=1}^{N} a_{a_{j}} \chi_{a_{j}}(x, y)+\phi\left(\sum_{j=1}^{N} a_{b_{j}} \chi_{b_{j}}(x, y)-\sum_{j=1}^{N} a_{a_{j}} \chi_{a_{j}}(x, y)\right)
$$

or in matrix form

$$
\begin{gathered}
f_{c}(x, y)=\left\{\chi_{a}(x, y)\right\}^{T}\left[\chi_{a}\right]^{-1}\{f\}+\phi\left(\left\{\chi_{b}(x, y)\right\}^{T}\left[\chi_{b}\right]^{-1}\{f\}-\left\{\chi_{a}(x, y)\right\}^{T}\left[\chi_{a}\right]^{-1}\{f\}\right) \\
\left.f_{c}(x, y)=\left[\left\{\eta_{a}\right\}^{T}+\phi\left(\left\{\eta_{b}\right)\right\}^{T}-\left\{\eta_{a}\right\}^{T}\right)\right]\{f\}
\end{gathered}
$$




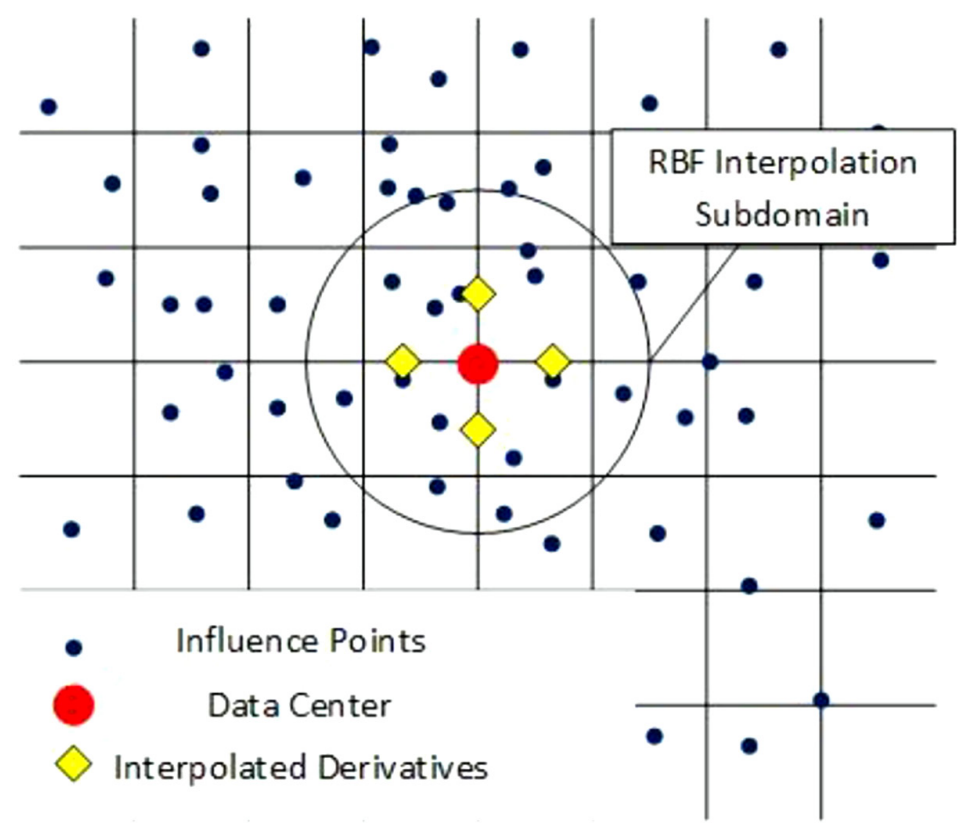

Figure 3: Topology for LRC-MM.

The same blending scheme described in this section can be used to approximate the derivatives as well and applied to solving a PDE. A sample point distribution with an influence topology including the data center and locations for derivative interpolation is shown in Fig. 3. The Localized RBF Collocation Meshless Method (LRC-MM) is used with the blending scheme to solve the inviscid Burgers' equation, 2-D linear advection equation, and the 2-D compressible Euler's equation to demonstrate the RBF blending scheme.

\section{ONE DIMENSIONAL INVISCID BURGERS EQUATION}

The solution of the 1-D inviscid Burgers equation is computed using the LRC-MM blended approach. This example illustrates the blended interpolation concept applied to the solution of a PDE with a shock or discontinuity. The governing equation and the initial condition are given below in eqn (26) while the plot of the solution evolution from 0 to 1 second is shown in Fig. 4.

$$
\begin{gathered}
\frac{\partial u(x, t)}{\partial t}+u \frac{\partial u(x, t)}{\partial x}=0 \\
u(x, 0)=1 \text { for } x<0.2 \\
u(x, 0)=0 \text { for } x \geq 0.2 \\
u(0, t)=1
\end{gathered}
$$



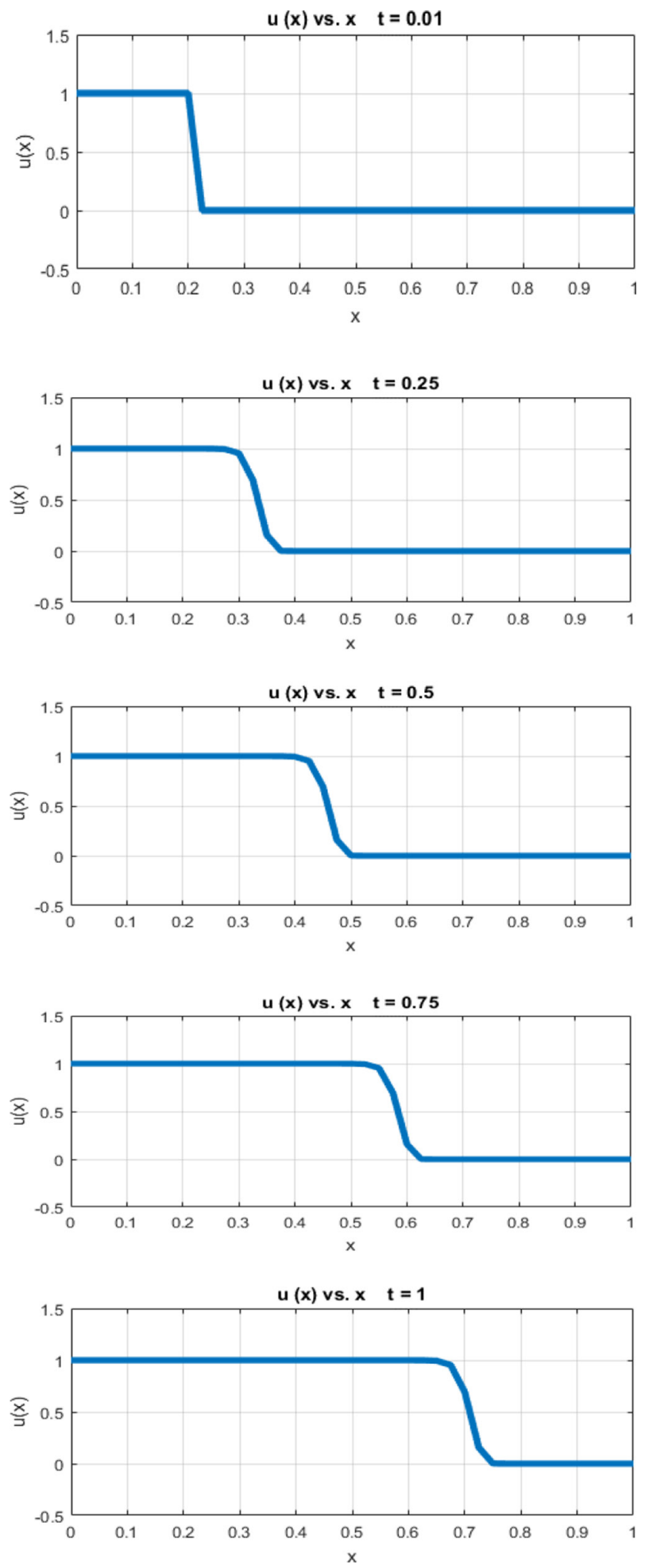

Figure 4: Results for the 1-D Burgers Equation using the blended LRC-MM. 


\section{TWO-DIMENSIONAL LINEAR ADVECTION EQUATION}

The solution of the 2-D linear advection equation is computed using the LRC-MM blended approach. This example illustrates the blended interpolation concept applied to the solution of a highly convective PDE. The governing equation, the initial and boundary conditions are given below in eqn (27). A contour plot of the velocity magnitude is shown in Fig. 5 where it is clearly observed how the solution becomes unstable without the blending of shape parameters.

$$
\begin{gathered}
\frac{\partial u}{\partial t}+U_{1} \frac{\partial u}{\partial x}+U_{2} \frac{\partial u}{\partial y}=0 \\
u(x, 0, t)=2 \text { for } x \leq 0.3 \\
u(x, 0, t)=0 \text { for } x \geq 0.3 \\
u(0, y, t)=2 \text { for } y \leq 0.3 \\
u(0, y, t)=0 \text { for } y \geq 0.3 \\
u(x, y, 0)=0
\end{gathered}
$$

where

$$
U_{1}=\frac{\sqrt{2}}{2}, \quad U_{2}=\frac{\sqrt{2}}{2}
$$

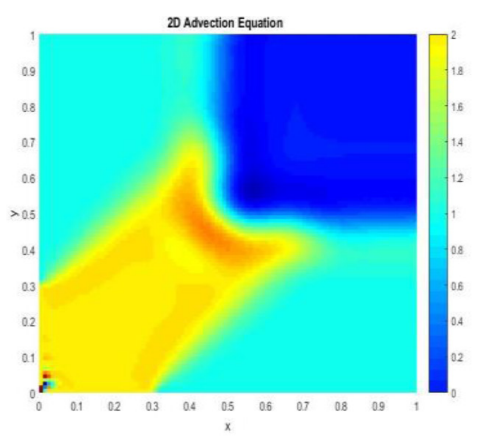

Unstable

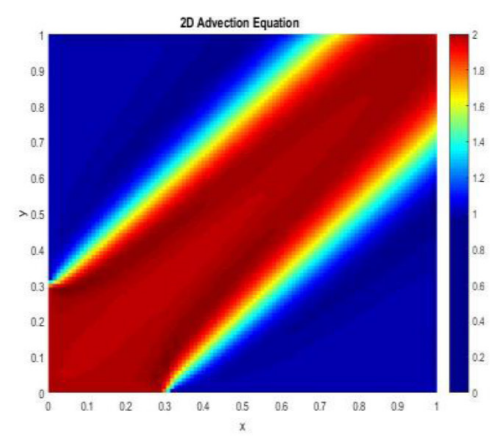

Stable

Figure 5: Constant shape parameter (Left) and blended shape parameter (Right) for diagonal wave problem.

\section{COMPRESSIBLE EULER EQUATIONS}

The compressible Euler equations are also solved using the LRC-MM blended approach. The compressible Euler equation is a non-linear hyperbolic PDE where discontinuities or shock waves can potentially manifest in the solution. The governing equations are shown below in eqn (28).

$$
\frac{\partial Q}{\partial t}+\frac{\partial F}{\partial x}+\frac{\partial G}{\partial y}=0
$$


where

$$
\begin{gathered}
Q=\left\{\begin{array}{c}
\rho \\
\rho u \\
\rho v \\
\rho e_{t}
\end{array}\right\} \quad F=\left\{\begin{array}{c}
\rho u \\
\rho u^{2}+P \\
\rho u v \\
\left(\rho e_{t}+P\right) u
\end{array}\right\} \quad G=\left\{\begin{array}{c}
\rho v \\
\rho u v \\
\rho v^{2}+P \\
\left(\rho e_{t}+P\right) v
\end{array}\right\} \\
e_{t}=e+\frac{1}{2}\left(u^{2}+v^{2}\right) \\
P=\rho(\gamma-1) e
\end{gathered}
$$

A flux vector splitting method is used to split the flux vectors into positive and negative components to upwind the equations. The split flux vector derivatives are interpolated either slightly upstream depending on the direction the up-winding needs to occur determined by the eigenvalues of the system.

A supersonic channel flow problem is chosen as an example to illustrate the solution of the compressible Euler equations using the blended LRC-MM scheme. The geometry and point distribution are shown in Fig. 6. The inlet Mach number is 3, inlet pressure is $100 \mathrm{kPa}$ and inlet temperature is $300 \mathrm{~K}$. The specific heat ratio is 1.4 and the gas constant is $287.05 \mathrm{~J} / \mathrm{kg} \mathrm{K}$. The geometry of the problem is $\mathrm{L}_{1}=10 \mathrm{~cm}, \mathrm{~L}_{2}=20 \mathrm{~cm}, \mathrm{~L}_{3}=30 \mathrm{~cm}$ and $\mathrm{H} 1=20 \mathrm{~cm}$ with a deflection angle of $10^{\circ}$ [12]. A steady state solution is obtained by marching through the domain using local time stepping. The contour plot of the Mach number of the LRC-MM solution is shown below in Fig. 7 as it compares with a Finite Differences Method (FDM) solution on a much more resolved point distribution.

The advantage of LRC-MM lies in its spectral convergence capabilities allowing high accurate interpolations in coarse distributions of scattered data points. In this case, the FDM solution shown in Figure 7 was generated in a $140 \times 100(14,000)$ node distribution, while the LRC-MM solution was generated in less than 1,000 data points. The goal for the future will be to use an adaptive refinement algorithm with the blended LRC-MM to automatically add points where steep gradients or discontinuities exist.

If a constant shape parameter, which yields high condition number, were to be used, the solution would become oscillatory and eventually unstable. This is shown in the Figure 8 where oscillations can be seen appearing near the ramp wall. This example demonstrates further the importance of blending the shape parameter for problems with steep gradients or discontinuities.

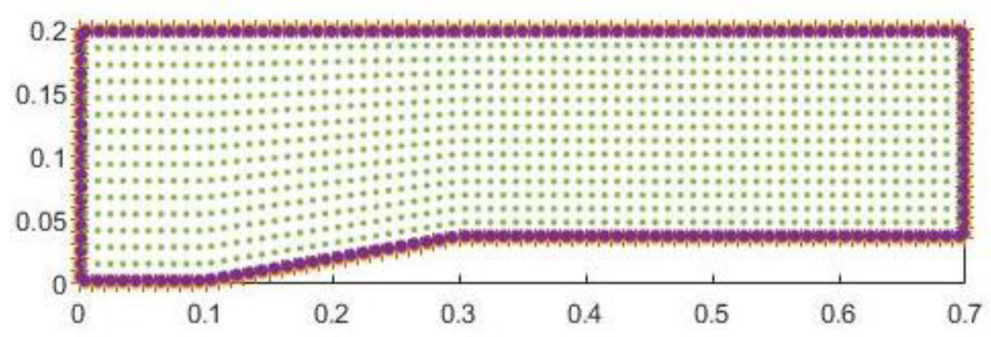

Figure 6: Problem domain and point distribution for blended LRC-MM. 

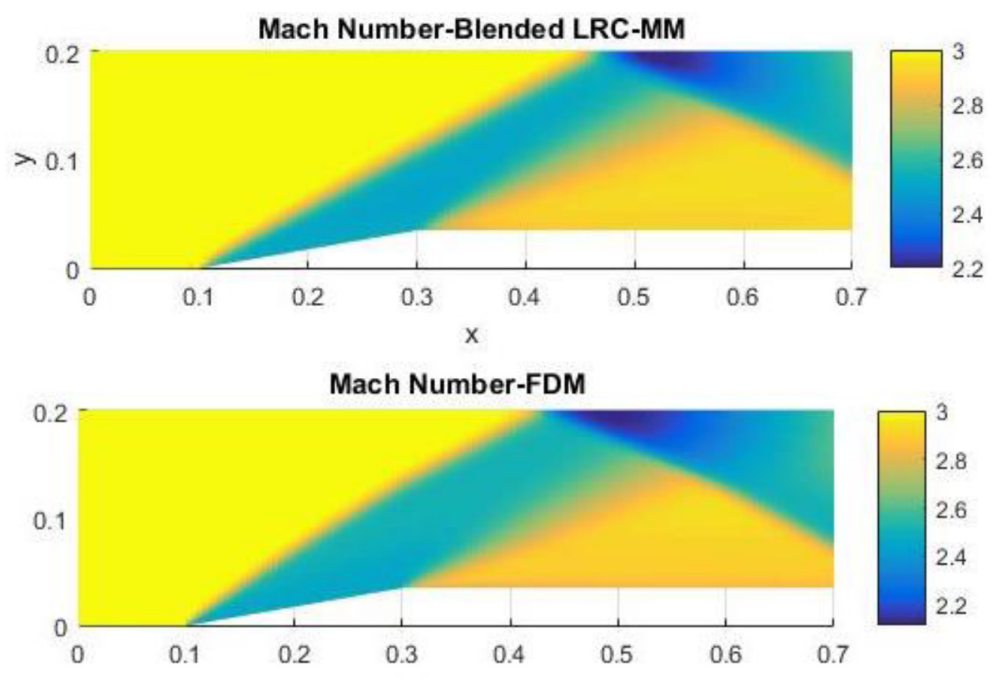

Figure 7: Mach Number comparison of the blended LRC-MM solution against finite difference method solution.

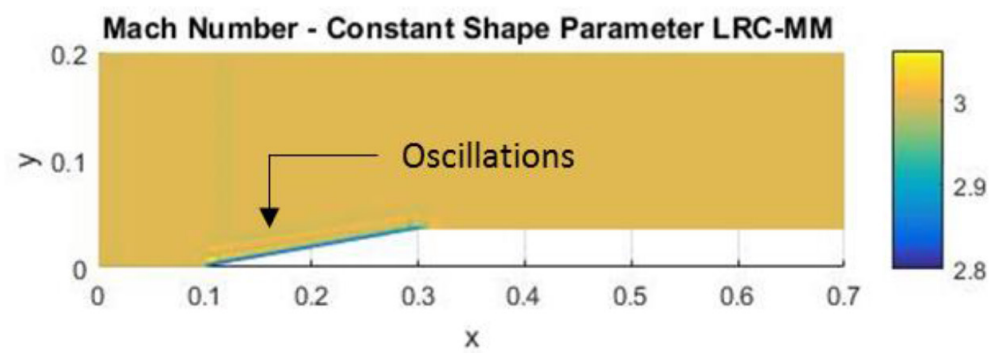

Figure 8: LRC-MM solution using constant high conditioning RBF interpolation.

\section{CONCLUSION}

The blended RBF interpolation approach coupled with the LRC-MM can solve hyperbolic PDEs. The blended RBF interpolation takes advantage of the low shape parameter and low conditioning when shocks are encountered allowing the computation to remain stable while effectively capturing the shock. When the solution is smooth, the blended RBF approach allows for high condition to be used to increase the order of the solution. The examples presented illustrate this notion by successfully solving the inviscid Burgers equation, two-dimensional linear advection and the compressible Euler equations.

\section{REFERENCES}

[1] Cheng, A.H.D., Golberg, M.A., Kansa, E.J. \& Zammito, G., Exponential convergence and H-c multiquadric collocation method for partial differential equations. Numerical Methods for Partial Differential Equation, 19, pp. 571-594, 2003.

https://doi.org/10.1002/num.10062 
[2] Sarler, B. \& Vertnik, R., Meshfree explicit local explicit radial basis function collocation method for diffusion problems. Computers \& Mathematics with Application, 51(8), pp. 1269-1282, 2005. https://doi.org/10.1016/j.camwa.2006.04.013

[3] Sarler, B., Tran-Cong, T. \& Chen, C.S., Meshfree direct and indirect local radial basis function collocation formulations for transport phenomena. Boundary Elements XVII, eds A. Kassab, C.A. Brebbia \& E. Divo, WIT Press, Southampton, UK, pp. 417-428, 2005.

[4] Gerace, S., Erhart, K., Kassab, A. \& Divo, E., A model-integrated localized collocation meshless method for large scale three dimensional heat transfer problems. Engineering Analysis, 45, pp. 2-19, 2014.

https://doi.org/10.1016/j.enganabound.2014.01.014

[5] Kelly, J, Divo, E. \& Kassab, A.J., Numerical solution of the two-phase incompressible navier-stokes equations using a GPU-Accelerated meshless method engineering analysis with boundary elements. Engineering Analysis, 40, pp. 36-49, 2014.

[6] Gerace, S., Erhart, K., Divo, E. \& Kassab, A., Adaptively refined hybrid FDM/Meshless scheme with applications to laminar and turbulent flows. CMES: Computer Modeling in Engineering and Science, 81(1), pp. 35-68, 2011.

[7] Erhart, K., Kassab, A.J. \& Divo, E., An inverse localized meshless technique for the determination of non-linear heat generation rates in living tissues. International Journal of Heat and Fluid Flow, 18(3), pp. 401-414, 2008. https://doi.org/10.1108/09615530810853655

[8] Divo, E.A. \& Kassab, A.J., An efficient localized RBF meshless method for fluid flow and conjugate heat transfer. ASME Journal of Heat Transfer, 129, pp. 124-136, 2007. https://doi.org/10.1115/1.2402181

[9] Divo, E.A. \& Kassab, A.J., Iterative domain decomposition meshless method modeling of incompressible flows and conjugate heat transfer. Engineering Analysis, 30(6), pp. 465-478, 2006. https://doi.org/10.1016/j.enganabound.2006.02.002

[10] Divo, E. \& Kassab, A.J., Localized meshless modeling of natural convective viscous flows. Numerical Heat Transfer, Part B: Fundamentals, 53, pp. 487-509, 2008. https://doi.org/10.1080/10407790802083190

[11] Harris, M., Kassab, A. \& Divo, E., Application of a RBF blending interpolation method to problems with shocks. Computer Assisted Methods in Engineering and Science, Institute of Fundamental Technological Research, Polish Academy of Science, 2015

[12] Hoffman, K.A. \& Chiang, S.T., Computational fluid dynamics volume 2. Engineering Education System, 2000.

[13] Pepper, D.W., Kassab, A.J. \& Divo, E.A., Introduction to Finite Element, Boundary Element, and Meshless Methods: With Application to Heat Transfer and Fluid Flow, ASME Press, 2014. https://doi.org/10.1115/1.860335

[14] Hirsch, C., Numerical Computation of Internal and External Flows Volume 2: Computational Methods for Inviscid and Viscous Flows, John Wiley \& Sons Ltd, 1984. 